\title{
Evaluation of discomfort and tolerability to bronchoscopy according to different sedation procedures with midazolam
}

\author{
TAKESHI MATSUMOTO, KOJIRO OTSUKA, RYOJI KATO, RYOKO SHIMIZU, \\ TAKEHIRO OTOSHI, DAICHI FUJIMOTO, TAKAHISA KAWAMURA, KOJI TAMAI, \\ KAZUMA NAGATA, KYOKO OTSUKA, ATSUSHI NAKAGAWA and KEISUKE TOMII
}

Department of Respiratory Medicine, Kobe City Medical Center General Hospital, Kobe, Hyogo 650-0047, Japan

Received July 21, 2014; Accepted March 20, 2015

DOI: $10.3892 / \mathrm{etm} .2015 .2547$

\begin{abstract}
Patients frequently experience great discomfort during a bronchoscopy for the diagnosis of lung neoplasms. Sedation is generally recommended during bronchoscopy; however, few studies have evaluated the discomfort and tolerability of patients to a bronchoscopy with regard to the administration procedures. The aim of the present study was to evaluate the discomfort and tolerability of patients undergoing a bronchoscopy using different sedation procedures with midazolam. The retrospective survey of sedation during bronchoscopy involved the comparison of two periods: January-March 2012 (first period) and July-September 2012 (second period). A numerical rating score, which ranged between 1 (best) and 5 (worst) according to the subjective view of the patients, was used to rate patient discomfort, pain, sensation, time and tolerability to the bronchoscopy. In the first period, $2.5 \mathrm{mg}$ midazolam was administered prior to the initiation of surgery, and additional doses of midazolam was added in 2.5-mg increments whenever the patient deviated from the target sedation level. In the second period, 2.0 or $3.0 \mathrm{mg}$ midazolam was administered prior to the initiation of surgery, and additional midazolam doses were administered in $1.0-\mathrm{mg}$ increments until the patients were sedated to the target sedation level. In total, 60 and 68 valid responses were obtained in the first and second periods, respectively. The patients in the second period exhibited significantly improved discomfort and pain scores during the bronchoscopy and higher rates of consent to re-examination, as compared with the patients in the first period $(1.89 \pm 1.40$ vs. $2.78 \pm 1.52, \mathrm{P}<0.001 ; 1.48 \pm 1.13$ vs. $2.00 \pm 1.37$, $\mathrm{P}=0.005 ; 2.45 \pm 1.62$ vs. $3.13 \pm 1.47, \mathrm{P}=0.013$, respectively). The
\end{abstract}

Correspondence to: Dr Takeshi Matsumoto, Department of Respiratory Medicine, Kobe City Medical Center General Hospital, 2-1-1 Minatojima-minamimachi, Chuo-ku, Kobe, Hyogo 650-0047, Japan

E-mail: fu_rinkazan0202@yahoo.co.jp

Key words: discomfort, tolerability, bronchoscopy, sedation, midazolam amount of midazolam administered was significantly higher in the second period. There were no fatal complications during the bronchoscopy in either period. In conclusion, the present study observed that the administration of additional midazolam in small doses, until the target sedation level is achieved, is a safe procedure that is associated with significantly less discomfort and pain during bronchoscopy and a greater consent to re-examination when compared with the administration of a fixed dose of midazolam.

\section{Introduction}

A bronchoscopy is the gold-standard diagnostic method for lung neoplasms (1). However, patients frequently experience severe discomfort during the procedure. As a result, numerous patients have recognized that a bronchoscopy is painful and difficult procedure, and avoid undergoing the examination if possible. This hesitation may lead to undesirable delays in treatment. Thus, more comfortable bronchoscopic procedures are necessary for early diagnosis and treatment of unknown pulmonary lesions.

A number of large surveys on bronchoscopic procedures have been performed (2-4) and numerous guidelines for bronchoscopy have been published worldwide $(1,5,6)$. In 2009 , the Japan Society for Respiratory Endoscopy performed a questionnaire survey on bronchoscopy in clinical practice to establish a guideline for painless bronchoscopy (7). In the survey, the discomfort and tolerability to a bronchoscopy were evaluated, and the results were significantly improved in patients who were sedated during the procedure, as compared with those who did not receive sedation.

Sedation during bronchoscopy is recommended in several guidelines $(1,5,6)$ and the administration of sedative agents is recommended in small doses. However, few studies have evaluated the discomfort and tolerability during a bronchoscopy with regard to the different administration protocols of sedatives. Thus, the precise advantages and disadvantages of small-dose administration of sedatives remain unknown. We have historically chosen fixed dose administration of midazolam to perform sedation during a bronchoscopy; however, the level of patient satisfaction is relatively poor. The patients surveyed were hesitant to repeat the bronchoscopy, potentially delaying their diagnosis and resulting in a 
poor prognosis. Therefore, the present study compared two midazolam administration protocols, and a numerical rating score was used to determine the level of patient satisfaction. The aim of the current study was to evaluate patient discomfort and tolerability to bronchoscopy following two different sedation procedures with midazolam.

\section{Materials and methods}

Patients. The study was a single-center retrospective survey of sedation during bronchoscopy. Two treatment periods were compared, namely January-March 2012 (first period) and July-September 2012 (second period), in which a different sedative procedure was performed. Consecutive patients who underwent a planned bronchoscopy were screened, and clinical data were collected from a questionnaire and the medical records of the patients. A blood test was performed to assess hepatic and renal function, and patients with levels of aspartate aminotransferase of $>100$ IU/1, alanine aminotransferase of $>100 \mathrm{IU} / 1$, total bilirubin of $>1.5 \mathrm{mg} / \mathrm{dl}$ and creatinin of $>1.5 \mathrm{mg} / \mathrm{dl}$ were excluded. In addition, patients who had chronic respiratory failure (percutaneous oxygen saturation of $<90 \%$ in room air) were excluded, as were those requiring procedures with intubation. Written informed patient consent for performing sedation during bronchoscopy was obtained from the patients and the study was approved by the Institutional Review Board of Kobe City Medical Center General Hospital (Kobe, Japan).

Questionnaire. A previously used questionnaire (7) was referred to for the construction of the questionnaire. Anonymous questionnaires were distributed to the bronchoscopists on the procedure day and to the patients, after they recovered from the sedation, on the same day and were concurrently analyzed. Various factors associated with the bronchoscopic procedures were evaluated in the questionnaire. For the bronchoscopists, a multiple choice survey format was utilized. Their questionnaire included patient age and gender, years of experience as a bronchoscopist, outpatient or inpatient status, use of pharyngeal anesthesia, route of bronchoscopy (nasal or oral), diameter of bronchoscope ( $<6$ or $>6 \mathrm{~mm}$ ), use of local anesthesia during bronchoscopy, amount of sedative agents administered, and time period of the procedure. For the patients, according to the subjective view of the patients, a numerical rating score that ranged between 1 (best) and 5 (worst) was used. Their questionnaire included the degree of discomfort, pain, sensation, time and tolerability to bronchoscopy (7). In addition, the questionnaire queried the memory of the bronchoscopy procedure. Complications during and 7 days after the bronchoscopy were evaluated. Complications included changes to the blood pressure, heart rate and oxygen saturation, prolonged sedation (defined as the need for additional antagonists above what is usually administered), pneumothorax, massive hemorrhage, pulmonary infection, bronchial asthma, respiratory failure, lidocaine intoxication, cardiovascular events, central airway obstruction and perforations. During bronchoscopy, oxygen therapy was performed for the temporal oxygen desaturation caused by insertion of bronchoscopy, minor bleeding or persistent cough. Lack of concordance between the bronchos- copist and patient questionnaire, or the absence of a response to a questionnaire item, invalidated that questionnaire item.

Bronchoscopy procedure. Prophylactic antibiotics were only administered to patients who were asplenic, had a heart valve prosthesis or had a previous history of endocarditis. Pharyngeal anesthesia with $4 \%$ lidocaine was performed prior to bronchoscopy. Premedication with atropine was not performed. In addition, intubation was not performed prior to bronchoscopy, with the exception of endobronchial ultrasound-guided transbronchial needle aspiration (EBUS-TBNA). Intravenous administration of midazolam (Astellas, Tokyo, Japan) was conducted during the bronchoscopy to sedate the patients; however, intravenous sedation was not performed on outpatients without an escort. During the bronchoscopy, $1 \%$ lidocaine was administered for local anesthesia of the bronchial mucosa. The blood pressure, heart rate, oxygen saturation, electrocardiogram and consciousness level of the patient were continually measured during the bronchoscopy procedure. When the oxygen saturation decreased, supplemental oxygen was provided to maintain an oxygen saturation of $>90 \%$. Following the bronchoscopy procedure, $0.25 \mathrm{mg}$ flumazenil (Astellas) was administered intravenously. Five factors were assessed in the observation room following the bronchoscopy, including whether the patient had clear consciousness, was able to walk unaided, had a stable respiratory rate, had the same preoperative blood pressure and had the same preoperative percutaneous oxygen saturation. Outpatients were subsequently discharged with a warning with regard to the potential for prolonged sedation. Additional administration of flumazenil was considered in the event of prolonged unconsciousness.

Midazolam sedation procedure. The midazolam administration procedure differed between the first and second periods (Fig. 1). In the first period, $2.5 \mathrm{mg}$ midazolam was administered once prior to the initiation of the bronchoscopy. Following the initiation of the bronchoscopy, midazolam was applied in 2.5-mg increments when the sedation level became $\geq 1$, according to the Richmond Agitation Sedation Scale (RASS) (8) but the interval time of additional administration was at least $2 \mathrm{~min}$. In the second period, 2.0 (mainly patients $>75$ years) or $3.0 \mathrm{mg}$ (mainly patients $<75$ years) midazolam was administered prior to bronchoscopy, and subsequent doses of midazolam were administered in 1.0-mg increments until the patients were sedated to the target sedation level (between -2 and -1 on the RASS). The interval time of additional administration was at least $2 \mathrm{~min}$. Following the initiation of the bronchoscopy, midazolam was administered in $1.0-\mathrm{mg}$ increments whenever the patient deviated from the target sedation level, and the interval time of additional administration was at least $2 \mathrm{~min}$.

Statistical analysis. Continuous variables are expressed as the mean \pm standard deviation, unless otherwise stated, and were compared using an unpaired Student's t-test or Wilcoxon rank sum test, as appropriate. Categorical variables were compared using the $\chi^{2}$ test or Fisher's exact test, as appropriate. $\mathrm{P}<0.05$ was considered to indicate a statistically significant difference. All statistical analyses were performed using JMP 8.0.2 software (SAS Institute, Inc., Cary, NC, USA). 
Table I. Characteristics of the patients and bronchoscopists during each period.

\begin{tabular}{|c|c|c|c|}
\hline Characteristics & First period $(n=60)$ & Second period $(n=68)$ & P-value \\
\hline Age $^{\mathrm{a}}$, years & $68.0 \pm 10.7$ & $67.3 \pm 11.5$ & 0.80 \\
\hline Gender (male/female), $\mathrm{n}$ & $30 / 30$ & $43 / 25$ & 0.13 \\
\hline \multicolumn{4}{|l|}{ Bronchoscopy procedures, n (\%) } \\
\hline 1 & $47(78)$ & $55(81)$ & 0.72 \\
\hline $2-5$ & $11(18)$ & $13(19)$ & 0.91 \\
\hline$\geq 6$ & $2(3)$ & $0(0)$ & 0.13 \\
\hline \multicolumn{4}{|l|}{ Years as a bronchoscopist, $\mathrm{n}(\%)$} \\
\hline 1 & $18(31)$ & $14(21)$ & 0.22 \\
\hline $2-5$ & $16(27)$ & $26(39)$ & 0.16 \\
\hline$\geq 6$ & $25(42)$ & $27(39)$ & 0.81 \\
\hline Outpatient/inpatient, $\mathrm{n}$ & $55 / 5$ & $68 / 0$ & 0.02 \\
\hline Pharyngeal anesthesia with $4 \%$ lidocaine ${ }^{\mathrm{a}}, \mathrm{ml}$ & $7.9 \pm 1.4$ & $7.8 \pm 1.9$ & 0.70 \\
\hline Route (nasal/oral), n & $42 / 13$ & $48 / 11$ & 0.51 \\
\hline Diameter of bronchoscope $(<6 />6 \mathrm{~mm}), \mathrm{n}$ & $56 / 4$ & $64 / 4$ & 1.0 \\
\hline Local anesthesia with $1 \%$ lidocaine $^{\mathrm{a}}, \mathrm{ml}$ & $10.5 \pm 3.3$ & $9.5 \pm 2.0$ & 0.45 \\
\hline \multicolumn{4}{|l|}{ Length of procedure, $\mathrm{n}(\%)$} \\
\hline $1-10 \min$ & $14(23)$ & $19(28)$ & 0.55 \\
\hline $11-20 \mathrm{~min}$ & $23(38)$ & $27(40)$ & 0.87 \\
\hline$\geq 21 \mathrm{~min}$ & $23(38)$ & $22(32)$ & 0.48 \\
\hline
\end{tabular}

${ }^{\text {aD }}$ ata are presented as the mean \pm standard deviation.

\section{First period}

First administration

\begin{tabular}{|c|}
\hline $\begin{array}{c}\text { Midazolam } \\
2.5 \mathrm{mg}\end{array}$ \\
\hline
\end{tabular}

Bronchoscopy started Added if needed

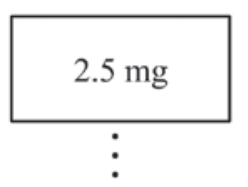

Figure 1. Midazolam administration protocol for each period.

\section{Results}

Study population. The first period included 115 patients who underwent a planned bronchoscopy. No patients were excluded following the results of the blood tests or oxygen saturation levels. However, a total of 7 patients underwent intubation with EBUS-TBNA and 6 patients did not wish to undergo sedation; therefore, 102 patients were eligible for the screening. Of these, 42 patients returned incomplete questionnaires. Thus, 60 valid responses were ultimately reviewed.
:

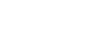

Second period

First administration

Midazolam

Added if needed

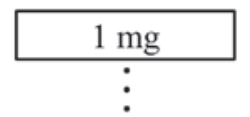

Bronchoscopy started Added if needed

$1 \mathrm{mg}$

: 


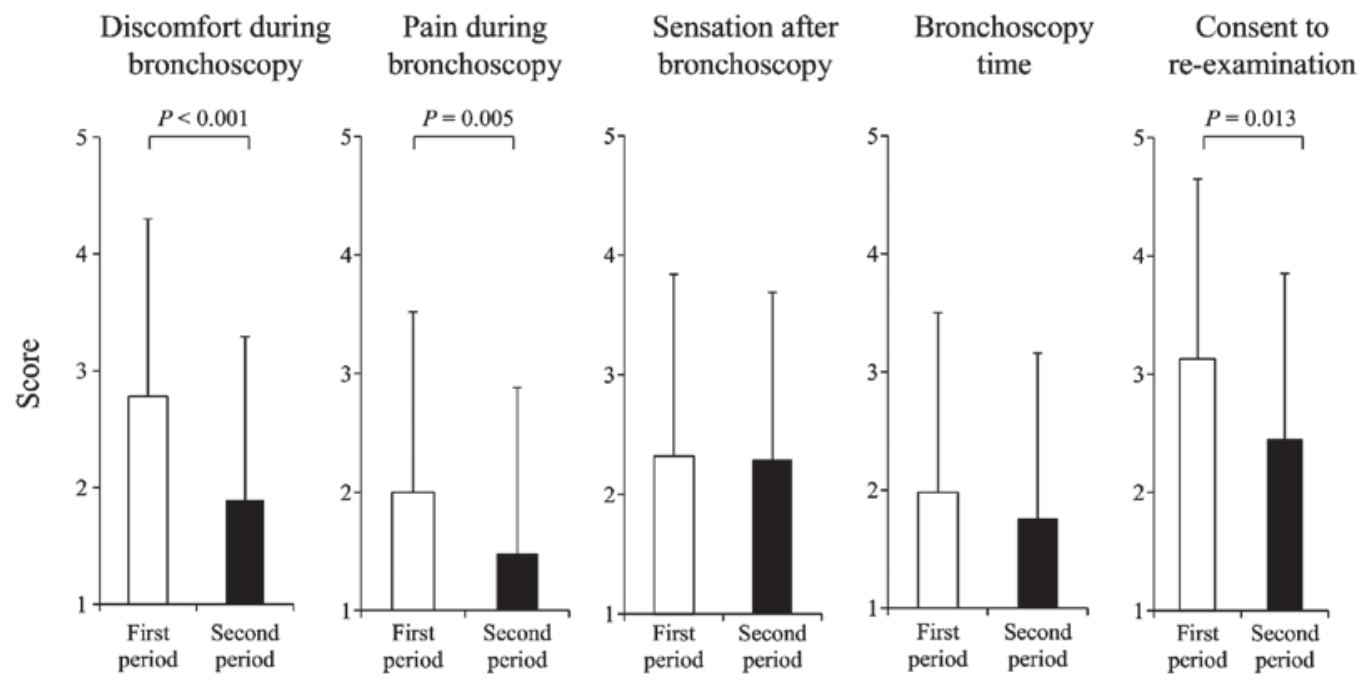

Figure 2. Patient scores during and following bronchoscopy in each period. The scores ranged between 1 (best) and 5 (worst). Data are expressed as the mean values and the bars represent standard deviation.

Table II. Complications during and following bronchoscopy in each period.

\begin{tabular}{lcc}
\hline Complications & $\begin{array}{c}\text { First period } \\
(\mathrm{n}=60)\end{array}$ & $\begin{array}{c}\text { Second period } \\
(\mathrm{n}=68)\end{array}$ \\
\hline $\begin{array}{l}\text { During bronchoscopy } \\
\text { Hypotension }\end{array}$ & $1(2)$ & $0(0)$ \\
Bradycardia & $1(2)$ & $0(0)$ \\
Prolonged sedation & $0(0)$ & $1(1)$ \\
Following bronchoscopy & & \\
Pneumonia & $2(3)$ & $1(1)$ \\
Pneumothorax & $1(2)$ & $0(0)$ \\
\hline
\end{tabular}

Data are presented as $\mathrm{n}(\%)$.

cantly less memory of the bronchoscopy, as compared with the patients treated in the first period (33 vs. $71 \%$; $\mathrm{P}<0.001)$.

Dose of midazolam. In total, 10/60 patients in the first period and 33/68 patients in the second period were administered additional midazolam following the first administration. The amount of midazolam used was significantly higher in the second period compared with the first period $(3.8 \pm 1.8$ vs. $3.0 \pm 1.3 \mathrm{mg} ; \mathrm{P}<0.001)$

Complications. There were no fatal complications during the bronchoscopy in either period. In the first period, there was one case of hypotension and one case of bradycardia that persisted during and following the bronchoscopy; however, these cases were improved by additional saline infusion. There were no cases of severe hypertension, hypotension, tachycardia or bradycardia, which required cessation, during the bronchoscopy in either period. In addition, there was no requirement for additional oxygen following the bronchoscopy in either period, even when oxygen therapy was performed during the bronchoscopy due to the temporal oxygen desaturation.
One case exhibited prolonged sedation in the second period. Within 7 days after the bronchoscopy, two cases of pneumonia and one case of pneumothorax were detected in the patients of the first period, while one case presented with pneumonia in the second period. In each case, treatment was successfully managed without the need for hospital admission (Table II).

\section{Discussion}

To the best of our knowledge, the present study was the first to evaluate the differences in the levels of patient discomfort and tolerability prior to and following different midazolam administration procedures during bronchoscopy. The study found that administration of additional midazolam in small doses until the target sedation level was achieved was a safe procedure that resulted in significantly less discomfort and higher tolerability, as compared with the administration of a fixed dose of midazolam.

A number of previous studies have recommended that sedation should be provided during a bronchoscopy. Jones et al recommended the use of midazolam and fentanyl for minor procedures since these drugs are available intravenously, have a rapid onset, and can be reversed by their antagonists (9). Midazolam administration for sedation procedures varies among studies; however, all studies are similar in administering midazolam in small doses. The British Thoracic Society guidelines recommend that midazolam is administered at an initial dose of 2.0-2.5 mg, followed by additional midazolam doses in $1.0-\mathrm{mg}$ increments as required after 2-10 min have passed (1). In addition, the European Respiratory Society recommends an initial dose of $2.0-2.5 \mathrm{mg}$, followed by additional doses in 1.0-mg increments after 2-5 min (5), while the American College of Chest Physicians recommends an initial dose of 0.06-0.07 mg/kg (6). Recently, an individualized midazolam-dosing protocol was reported. The results indicated that the loading dose should be $0.075 \mathrm{mg} / \mathrm{kg}$ for men aged $<65$ years and women aged $<70$ years, while a dose of $0.05 \mathrm{mg} / \mathrm{kg}$ should be applied to men aged $>66$ years and women aged $>71$ years, with subsequent doses of one-half the 
loading dose to be administered every $20 \mathrm{~min}$ (10). There are several guidelines and randomized control trials with regard to midazolam administration; however, to the best of our knowledge, no studies have evaluated patient discomfort and tolerability according to the administration procedure used, including the small-dose administration procedure.

The aim of the present study was to evaluate the differences in patient levels of satisfaction with changes in the sedation protocol. Midazolam is a water-soluble benzodiazepine with an elimination half-life of $\sim 2 \mathrm{~h}$. The drug targets $\gamma$-aminobutyric acid receptors in the brain, subsequently reducing anxiety and causing amnesia. Sedation is accomplished by adjusting the amount of midazolam. Flumazenil is an antagonist of midazolam that can reverse the effect of midazolam when overdosed (11).

However, there are certain negative results associated with sedation with midazolam. A randomized control study demonstrated that sedation with midazolam did not make the patients comfortable or willing to undergo a repeated bronchoscopy (12); however, the authors stated that the small dose of midazolam may have accounted for this effect. This observation appears to be in accordance with the results of the present study, in that the period in which additional midazolam was administered resulted in improved patient tolerance in comparison with the other period. Furthermore, several studies have reported the effects of sedatives with the addition of opioids, including fentanyl, on bronchoscopy $(9,13)$. However, as fentanyl is an opioid, the treatment must be strictly managed; thus, use of fentanyl routinely in bronchoscopy is difficult. The present study demonstrated that a single administration of midazolam was sufficient to perform a bronchoscopy.

The patient scores for discomfort and pain during the bronchoscopy, and consent to re-examination were shown to improve in the second period, as compared with the first period. Thus, the second protocol appears to have been associated with reduced pain and a more comfortable bronchoscopy procedure, resulting in patients being more willing to undergo a repeated bronchoscopy. Therefore, administration of additional midazolam in small doses, until the target sedation level is achieved, can be recommended based on the improved scores obtained during the bronchoscopy in the present study. In addition, the results of the present study exhibited greater improvement in comparison to those of a nationwide study in Japan that used the same scoring system (7). In the nationwide study, the scores for discomfort, pain, sensation, time and tolerability among the sedated patients were $2.21 \pm 1.34$ (compared with $1.89 \pm 1.40$ in the second period of the present study), $1.53 \pm 0.92(1.48 \pm 1.13)$, $2.33 \pm 1.16(2.29 \pm 1.11), 2.22 \pm 1.28(1.76 \pm 1.18)$ and $2.68 \pm 1.49$ (2.45 \pm 1.62$)$, respectively. Midazolam and pethidine were used for sedation; however, the exact amounts of the sedative agents used are unknown. The improved results obtained in the present study may be associated with the higher amount of midazolam administered, since the use of sedative agents and sufficient sedation is not common practice in Japan (14). This observation indicates the importance of the administration of adequate amounts of sedative agents. Although patients in the second period had significantly less memory of the bronchoscopy, the scores for sensation following the bronchoscopy and perception of the bronchoscopy time did not significantly differ between the two periods. Thus, these results may not have been closely associated with memory during the bronchoscopy.

Complications during and following the bronchoscopy were also reviewed in the present study, and there were no fatal complications. A previous nationwide study reported diagnostic bronchoscopy complication rates of $0.17-1.93 \%$ and a mortality rate of $0.004 \%$ (15). The complications observed in the present study were minor and mild, and the majority of the patients were free from complications. In total, 4 patients developed pneumonia or pneumothorax within 7 days after the bronchoscopy; however, all the patients were successfully managed without the need for hospital admission. Therefore, careful monitoring during the procedure can ensure the safety of bronchoscopy with adequate sedation.

However, there are several limitations to the current study. Firstly, the study was conducted in two periods. Each period was 3 months in duration, which is relatively short; therefore, there may be several biases in the selection of patients and bronchoscopists. Furthermore, although all consecutive patients were screened, certain patients were excluded due to the return of incomplete questionnaires; therefore, it was possible that another selection bias occurred. However, the patient characteristics were not significantly different between the two periods, with the exception of the increased number of inpatients in the first period. As sedation was performed with the same protocol regardless of whether the patients were outpatients or inpatients, the difference may not have substantially affected the study results. The characteristics of the bronchoscopists were also not significantly different between the two periods; therefore, selection bias was minimized. Secondly, $21-31 \%$ of the bronchoscopists had $<1$ year experience performing bronchoscopies; however, in these cases, attending doctors provided supervision during bronchoscopies, and there were few problems accordingly.

In conclusion, the present study found that the administration of additional midazolam in small doses, until the target sedation level is achieved, is a safe procedure that results in significantly reduced patient discomfort and a higher tolerability when compared with the administration of a fixed dose of midazolam. Therefore, it would be better to administer additional midazolam in small doses, and in the future, the use of other sedatives or a combination of multiple sedatives must be discussed for further comfortable bronchoscopy.

\section{References}

1. Du Rand IA, Blaikley J, Booton R, et al; British Thoracic Society Bronchoscopy Guideline Group: British Thoracic Society guideline for diagnostic flexible bronchoscopy in adults: Accredited by NICE. Thorax 68 (Suppl 1): i1-i44, 2013.

2. Prakash UB, Offord KP and Stubbs SE: Bronchoscopy in North America: The ACCP survey. Chest 100: 1668-1675, 1991.

3. Honeybourne D and Neumann CS: An audit of bronchoscopy practice in the United Kingdom: A survey of adherence to national guidelines. Thorax 52: 709-713, 1997.

4. Smyth CM and Stead RJ: Survey of flexible fibreoptic bronchoscopy in the United Kingdom. Eur Respir J 19: 458-463, 2002.

5. Jose RJ, Shaefi S and Navani N: Sedation for flexible bronchoscopy: current and emerging evidence. Eur Respir Rev 22: 106-116, 2013.

6. Wahidi MM, Jain P, Jantz M, et al: American College of Chest Physicians consensus statement on the use of topical anesthesia, analgesia and sedation during flexible bronchoscopy in adult patients. Chest 140: 1342-1350, 2011. 
7. Fujisawa T, Kaneko N, Ikeda N, et al: Proposal for safe bronchoscopy which considers patient satisfaction based on a bronchoscopic examination questionnaire. Kikanshigaku 33: 3-11, 2011 (In Japanese).

8. Sessler CN, Gosnell MS, Grap MJ, et al: The Richmond Agitation-Sedation Scale: Validity and reliability in adult intensive care unit patients. Am J Respir Crit Care Med 166: 1338-1344, 2002.

9. Jones DR, Salgo P and Meltzer J: Videos in clinical medicine Conscious sedation for minor procedures in adults. N Engl J Med 364: e54, 2011.

10. Ogawa T, Imaizumi K, Hashimoto I, et al: Prospective analysis of efficacy and safety of an individualized-midazolam-dosing protocol for sedation during prolonged bronchoscopy. Respir Investig 52: 153-159, 2014.

11. Morgan GE, Mikhail MS and Murray MJ (eds): Clinical Anesthesiology. 3rd edition. McGraw-Hill, New York, NY, 2001 pp160-164.
12. Hatton MQ, Allen MB, Vathenen AS, Mellor E and Cooke NJ: Does sedation help in fibreoptic bronchoscopy? BMJ 309: 1206-1207, 1994.

13. Dreher M, Ekkernkamp E, Storre JH, Kabitz HJ and Windisch W: Sedation during flexible bronchoscopy in patients with pre-existing respiratory failure: Midazolam versus Midazolam plus Alfentanil. Respiration 79: 307-314, 2010.

14. Asano F, Aoe M, Ohsaki Y, et al. Bronchoscopic practice in Japan: A survey by the Japan Society for Respiratory Endoscopy in 2010. Respirology 18: 284-290, 2013.

15. Asano F, Aoe M, Ohsaki Y, et al: Deaths and complications associated with respiratory endoscopy: A survey by the Japan Society for Respiratory Endoscopy in 2010. Respirology 17: 478-485, 2012. 